\title{
Assessment and Management of Over-Activity and Sleep Disorder in Mucopolysaccharidoses
}

\author{
Dougal Julian Hare ${ }^{1}$ Louise Mahon ${ }^{2}$ Stewart Rust ${ }^{3}$ \\ ${ }^{1}$ School of Psychology, Cardiff University, Cardiff, Wales, \\ United Kingdom \\ 2 Manchester Mental Health and Social Care NHS Trust, Manchester, \\ United Kingdom \\ 3 Department of Paediatric Psychosocialogy, Royal Manchester \\ Children's Hospital, Manchester, United Kingdom
}

\begin{abstract}
Address for correspondence Dr. Dougal Julian Hare, DClinPsy, School of Psychology, Cardiff University, Cardiff, Wales, United Kingdom (e-mail: HareD@cardiff.ac.uk).
\end{abstract}

J Child Sci 2018;8:e124-e127.

\begin{abstract}
Keywords

- mucopolysaccharides

- Sanfilippo syndrome

- sleep

- circadian

- activity

There is a growing awareness, based on both research and clinical studies, that abnormal sleep and circadian functioning are associated with the various forms of mucopolysaccharidoses (MPS), with sleep respiratory problems seemingly common in many forms of MPS. In the case of MPS III, there is evidence of syndrome-specific sleep disturbance related to an abnormal and possibly unique circadian rhythm. The presence of such patterns of sleep and activity has detrimental consequences for both the person with MPS and also invariably for her/his family and carers. On the basis of current evidence, routine assessment of sleep functioning is clearly warranted for all children with MPS III together with appropriate medical and behavioral interventions as required.
\end{abstract}

\section{Introduction}

The mucopolysaccharide (MPS) disorders are a group of lysosomal storage disorders in which the enzymes responsible for the catabolism of glycosaminoglycans (GAGs, previously known as MPS) are deficient. This deficiency results in the accumulation of GAGs in the cells, blood, and connective tissue of the body and brain. Seven distinct types of MPS disorders have been identified associated with different enzyme deficiencies and are usually, but not wholly, characterized by a period of normal development followed by physical and/or cognitive decline and often premature death. ${ }^{1}$ MPS I (Hurler syndrome and the less severe variants of Scheie and Hurler-Scheie syndromes) is particularly associated with skeletal abnormalities, motor and cognitive delay and occurs in approximately 1 in 100,000 live births although, as with all the MPS disorders, international variation exists. ${ }^{2}$ MPS II (Hunter syndrome) is associated almost entirely with males and occurs in approximately 1 in 170,000

received

October 25, 2017 accepted after revision April 18, 2018
Issue Theme Advances in Mucopolysaccharidoses; Guest Editors: Susanne G. Kircher, MD, PhD, MBA, Adriana M. Montaño, $\mathrm{PhD}$, and Christina Lampe, MD. live births with affected children identified as having a severe or mild variant phenotype depending on cognitive impairment and progression of the disorder. ${ }^{3}$ MPS III (Sanfilippo syndrome) is the most common MPS disorder, occurring in 1 out of 24,000 live births and with four genetically distinct subtypes (A-D) related to deficiencies in specific enzymes responsible for the breakdown of heparan sulfate. Other forms of MPS, including MPS IV (Morquio syndrome), MPS VI (Maroteaux-Lamy syndrome), MPS VII (Sly syndrome), and MPS IX (Natowicz syndrome), are extremely rare and tend to be associated with physical abnormalities rather than cognitive impairment.

When examining the clinical implications of the MPS disorders, researchers and clinicians are primarily concerned with the behavioral phenotype of a given disorder, which can be understood as the increased likelihood of specific behaviors being associated with any given disorder. ${ }^{4}$ Moreover, many children with MPS show signs of other developmental disabilities such as autism in MPS III, $^{5}$ as well as significant

\section{Copyright $\odot 2018$ Georg Thieme Verlag License terms KG Stuttgart · New York}


behavioral and emotional difficulties as part of their behavioral phenotype, ${ }^{6}$ the consequences of which are often exacerbated as many clinicians have no knowledge or experience of the underlying MPS or associated phenotype. Considering the various MPS disorders, sleep disturbance and fearfulness are features of the behavioral phenotypes for MPS I and MPS II presentation, with relatively normal development and few or no behavioral problems in the mild form of MPS II, while those with the severe form show behavioral problems, delayed speech, delayed development, and limited motor function. In contrast, the clinical presentation of MPS III is very heterogeneous ${ }^{7}$ with sleep disturbance and frequent behavioral problems relating to aggression, hyperactivity, orality, unusual emotional responses, and temper tantrums being identified via systematic review. ${ }^{6}$ Empirical studies of the behavioral phenotype for children in the middle phase (2-9 years) of MPS III $^{8}$ have subsequently identified elevated rates of hyperactivity, orality, bodily movements, and attentional problems as well as sleep difficulties. In contrast, children with MPS IV show distinct physical features with impaired mobility and speech but few behavior problems, these being primarily emotional and attentional $^{9}$

\section{Sleep and Circadian Rhythm Abnormalities in Mucopolysaccharidoses}

The association between sleep dysfunction and the MPS disorders, and in MPS III in particular, has long been noted by clinicians. ${ }^{10}$ A systematic review ${ }^{11}$ of sleep disorders associated with genetic disorders, including MPS, identified several studies using objective assessment such as polysomnography (PSG) and actigraphy but with mixed samples of children with MPS. PSG-based studies by Nashed et al and Santamaria et al ${ }^{12,13}$ with mixed MPS samples of four MPS IH, two MPS IHS, three MPS II, two MPS IV,two MPS IS, three MPS II, one MPS IIIB, four MPS IVA, one MPS VI, respectively, indicated normal sleep latency (i.e., time to falling asleep) but reduced overall sleep time. Both of these samples were selected on the basis of preexisting sleep-respiratory problems, and further work ${ }^{14}$ also utilizing a mixed sample (3 MPS I, 15 MPS II, 1 MPS III, 1 MPS IV, 4 MPS VI) but selected on the basis of MPS diagnosis rather than sleep or breathing difficulties, and focused on sleep architecture rather than sleep timing, identified that all children with MPS showed objective signs of obstructive sleep apnea.

All of the studies described above have used highly heterogeneous samples of children with MPS without any form of non-MPS comparison group. Turning to studies using more homogenic samples, a study ${ }^{15}$ using 48 -hour PSG with six children with MPS IIIA only (mean aged 14.1 years), and compared with age- and sex-matched controls, found impoverished total night-time sleep (240.5 minutes versus 458.3 minutes), but increased daytime sleep ( 88.8 minutes versus 24.8 minutes) with substantially decreased rapid eye movement sleep ( 8.03 versus $21.53 \%$ ). Overall, the overwhelming majority of MPS-related sleep research has focused on MPS III and a wide range of sleep problems, including bedtime resistance, sleep onset delay, sleep dura- tion, sleep anxiety, night waking, night behaviors, parasomnias, sleep disordered breathing, and daytime sleepiness, have long been identified as being part of the clinical presentation and are now regarded as part of the behavioral phenotype of MPS III. ${ }^{16,17}$ Such sleep disturbance has a major impact on individuals with MPS III and inevitably also on their families, with parents reporting disruption in their own sleep patterns and that of siblings. ${ }^{18}$ Such sleep difficulties are also associated with daytime challenging behavior ${ }^{16}$ and there is a trend for sleep difficulties, particularly sleep onset latency and sleep efficiency, to worsen with age and disease progression. ${ }^{17}$

There is emerging evidence that these sleep problems may be linked to an underlying disturbance in circadian functioning in MPS III, ${ }^{17,19,20}$ which may also be an influence on aggressive behavior and hyperactivity. ${ }^{21}$ The recent study by Mahon et al ${ }^{17}$ utilizing both actigraphy and melatonin assay reported that children with middle phase MPS III had higher endogenous melatonin concentrations between $6 \mathrm{am}$ and $8 \mathrm{am}$ and lower levels at $10 \mathrm{pm}$-midnight compared with age-matched controls. Moreover, there was effectively no significant differences in melatonin across 24 hours in the MPS III group, thus demonstrating a significantly abnormal circadian rhythm of melatonin concentration (-Fig. 1). This has clear implications for sleep function, as reduced levels of melatonin at night will result in difficulties falling asleep, while elevated levels of melatonin in the morning will lead to an increase in daytime napping. 22

Future work on circadian rhythm in middle phase MPS III $^{21}$ has examined fragmentation of rhythm (intradaily variability), stability of rhythm to external zeitgebers (interdaily stability), time of the "best fit" of circadian cycle (periodicity) and the onset of the least active 5-hour (L5 onset), and most active 10hour (M10 onset) periods as well as measuring activity levels across four quadrants of a 24-hour period (midnight-6 am, 6 am-12 pm, 12 pm-6 pm, and 6 pm-midnight). Circadian rhythms were found to be more fragmented and less stable in children with middle phase MPS III together with a later onset of wakefulness and activity and variability in the onset of the least active period. With regard to activity, children with MPS III demonstrated increased activity in the early morning hours (midnight- $6 \mathrm{am}$ ), followed by lower activity between 6 am and12 pm (-Fig. 2).

Overall, these data corroborate the earlier biochemical data and are indicative of a possibly unique pattern of circadian functioning associated with the middle stage of MPS III. These data have important clinical implications, as being "out of sync" with both the immediate environment and the circadian patterns of carers, family and society in general are likely to have a major impact on quality of life for children with MPS III and their families.

\section{Clinical Implications of Circadian and Sleep Abnormalities in MPS III}

Given the clinical and research evidence reviewed above, it is strongly recommended that at least all children with MPS III should be investigated for both sleep and circadian disturbance 


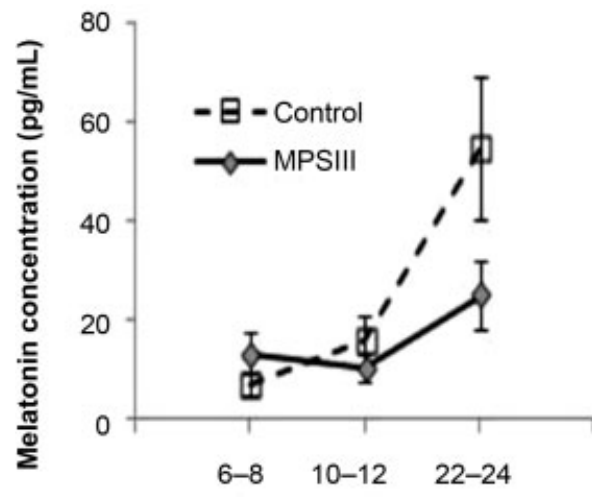

A

Time (hr)

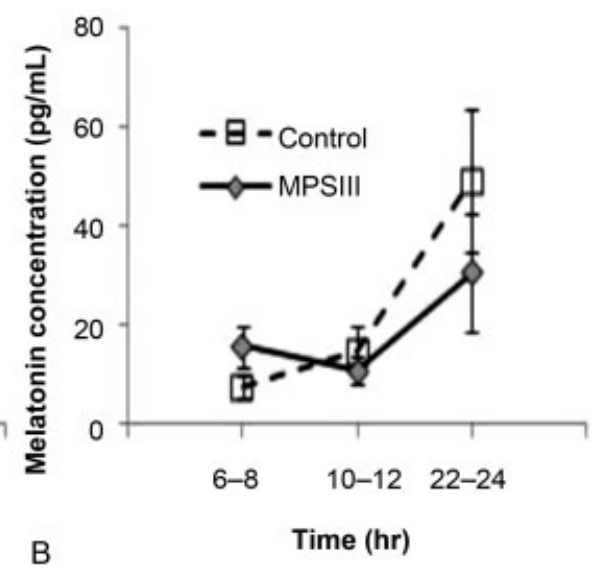

Fig. 1 Melatonin concentrations (average \pm standard error of mean [SEM]) in mucopolysaccharidosis III (MPS III) and control groups on (A) first day of actigraphic monitoring and (B) last day of actigraphic monitoring.

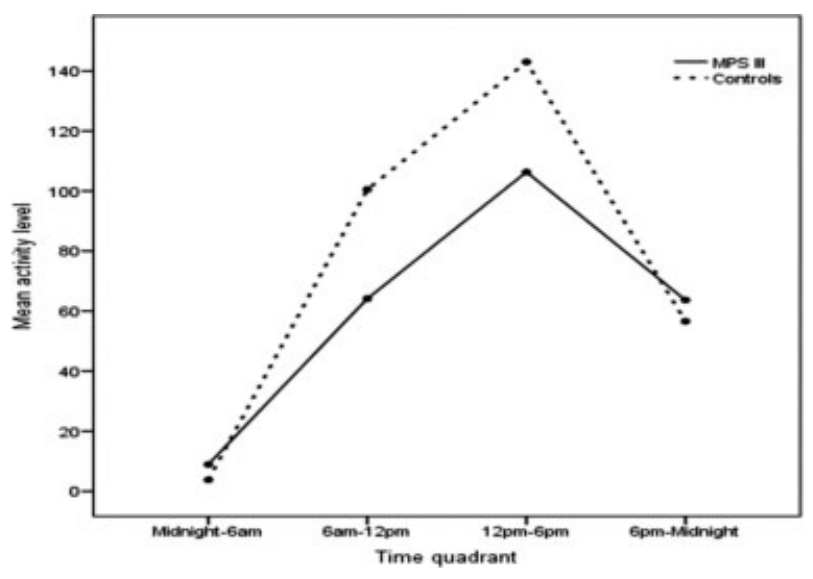

Fig. 2 Averaged activity levels for mucopolysaccharidosis (MPS III) and controls across 24-hour period.

as soon as possible after initial diagnosis and as part of the routine clinical management of the condition. In particular, both actigraphic assessment and melatonin assays have been shown to be feasible for children with MPS III to tolerate and for their families to manage. ${ }^{17,21}$ Moreover, behavioral interventions in particular have considerable utility for managing sleep problems in children with severe developmental disabilities $^{23,24}$ and it is recommended that following objective sleep assessment, advice on behavioral modification should be provided to caregivers with a specific focus on bedtime resistance, sleep onset difficulties, sleep anxiety, night-time waking, disruptive/dangerous behaviors at night, daytime sleep and tiredness. In addition, it is important for clinicians to be aware that sleep disturbance and dysfunction always affect more than just the child with MPS III and therefore to offer support for parents and siblings at the same time. With regard to physical and pharmacological interventions, upper airway assessment and management are currently recommended in the case of MPS I and MPS IV ${ }^{25,26}$ and clinicians should be alert to the possibility of this in the other forms of MPS. The evidence regarding pharmacological interventions for sleep disturbance in MPS is more limited, but the use of melatonin is supported by clinical ${ }^{27}$ as well as research studies, ${ }^{17}$ while the use of major tranquilizers with patients with MPS III is contraindicated given the risk of extrapyramidal symptoms. ${ }^{28}$ In addition to the specific issues identified for MPS I, III, and IV, it is also important for clinicians to be aware of that sleep dysfunction is also reported, albeit to a lesser degree, in other forms of MPS and that it would be advisable to routinely assess sleep in the course of ongoing clinical management.

A further implication of the recent research into sleep and circadian rhythm dysfunction in MPS III is the possibility of these forming part of the outcome variables in treatment trials, the more so given the very robust nature of actigraphic and melatonin assay data.

\section{References}

1 Udwin O, Dennis J. Psychological and behavioural phenotypes of genetically determined syndromes: a review of research findings. In: O'Brien G, Yule W, eds. Behavioural Phenotypes. Cambridge, UK: Cambridge University Press; 1995:35-44

2 Brown MB, Trivette PS. Mucopolysaccharide disorders. In: Phelps L, ed. Health-Related Disorders in Children and Adolescents: A Guidebook for Understanding and Educating. Washington DC: American Psychological Association; 1998:442-452

3 Wraith JE, Scarpa M, Beck M, et al. Mucopolysaccharidosis type II (Hunter syndrome): a clinical review and recommendations for treatment in the era of enzyme replacement therapy. Eur J Pediatr 2008;167(03):267-277

4 Dykens EM. Measuring behavioral phenotypes: provocations from the "new genetics". Am J Ment Retard 1995;99(05):522-532

5 Wolfenden C, Wittkowski A, Hare DJ. Symptoms of autism spectrum disorder (ASD) in individuals with mucopolysaccharide disease type III (Sanfilippo syndrome): asystematic review. J Autism Dev Disord 2017;47(11):3620-3633

6 Cross EM, Hare DJ. Behavioural phenotypes of the mucopolysaccharide disorders: a systematic literature review of cognitive, motor, social, linguistic and behavioural presentation in the MPS disorders. J Inherit Metab Dis 2013;36(02):189-200

7 Valstar MJ, Ruijter GJ, van Diggelen OP, Poorthuis BJ, Wijburg FA. Sanfilippo syndrome: a mini-review. J Inherit Metab Dis 2008;31 (02):240-252 
8 Cross EM, Grant S, Jones S, et al. An investigation of the middle and late behavioural phenotypes of Mucopolysaccharidosis Type-III. J Neurodev Disord 2014;6(01):46

9 Davison JE, Kearney S, Horton J, Foster K, Peet AC, Hendriksz CJ. Intellectual and neurological functioning in Morquio syndrome (MPS IVa). J Inherit Metab Dis 2013;36(02):323-328

10 Bax MC, Colville GA. Behaviour in mucopolysaccharide disorders. Arch Dis Child 1995;73(01):77-81

11 Mahon L. Objective Assessment of Sleep in Neurodevelopmental Disorders: A Study of Children with Mucopolysaccharidosis Type III [DClinPsy. Thesis]. Manchester, UK: University of Manchester; 2012

12 Nashed A, Al-Saleh S, Gibbons J, et al. Sleep-related breathing in children with mucopolysaccharidosis. J Inherit Metab Dis 2009; 32(04):544-550

13 Santamaria F, Andreucci MV, Parenti G, et al. Upper airway obstructive disease in mucopolysaccharidoses: polysomnography, computed tomography and nasal endoscopy findings. J Inherit Metab Dis 2007;30(05):743-749

14 Lin HY, Chen MR, Lin CC, et al. Polysomnographic characteristics in patients with mucopolysaccharidoses. Pediatr Pulmonol 2010; 45(12):1205-1212

15 Mariotti P, Della Marca G, Iuvone L, et al. Sleep disorders in Sanfilippo syndrome: a polygraphic study. Clin Electroencephalogr 2003;34(01):18-22(electroencephalography)

16 Fraser J, Gason AA, Wraith JE, Delatycki MB. Sleep disturbance in Sanfilippo syndrome: a parental questionnaire study. Arch Dis Child 2005;90(12):1239-1242

17 Mahon LV, Lomax M, Grant S, et al. Assessment of sleep in children with mucopolysaccharidosis type III. PLoS One 2014;9(02): e84128

18 Colville GA, Watters JP, Yule W, Bax M. Sleep problems in children with Sanfilippo syndrome. Dev Med Child Neurol 1996;38(06): 538-544
19 Canal MM, Wilkinson FL, Cooper JD, Wraith JE, Wynn R, Bigger BW. Circadian rhythm and suprachiasmatic nucleus alterations in the mouse model of mucopolysaccharidosis IIIB. Behav Brain Res 2010;209(02):212-220

20 Guerrero JM, Pozo D, Diaz-Rodriguez JL, Martinez-Cruz F, VelaCampos F. Impairment of the melatonin rhythm in children with Sanfilippo syndrome. J Pineal Res 2006;40(02):192-193

21 Mumford RA, Mahon LV, Jones S, Bigger B, Canal M, Hare DJ. Actigraphic investigation of circadian rhythm functioning and activity levels in children with mucopolysaccharidosis type III (Sanfilippo syndrome). J Neurodev Disord 2015;7(01):31

22 Lack LC, Bootzin RR. Circadian rhythm factors in insomnia and their treatment. In Perlis ML, Lichstein KL, eds. Treating Sleep Disorders: Principles and Practice of Behavioral Sleep Medicine. Hobocken, NJ: John Wiley \& Son; 200:305-343

23 Spruyt K, Curfs LMG. Non-pharmacological management of problematic sleeping in children with developmental disabilities. Dev Med Child Neurol 2015;57(02):120-136

24 Priday LJ, Byrne C, Totsika V. Behavioural interventions for sleep problems in people with an intellectual disability: a systematic review and meta-analysis of single case and group studies. J Intellect Disabil Res 2017;61(01):1-15

25 Muenzer J, Wraith JE, Clarke LA; International Consensus Panel on Management and Treatment of Mucopolysaccharidosis I. Mucopolysaccharidosis I: management and treatment guidelines. Pediatrics 2009;123(01):19-29

26 Giugliani R, Harmatz P, Wraith JE. Management guidelines for mucopolysaccharidosis VI. Pediatrics 2007;120(02):405-418

27 Malm G, Månsson JE. Mucopolysaccharidosis type III (Sanfilippo disease) in Sweden: clinical presentation of 22 children diagnosed during a 30-year period. Acta Paediatr 2010;99(08):1253-1257

28 Tchan MC, Sillence D. Extrapyramidal symptoms and medication use in Mucopolysaccharidosis type III.J Intellect Dev Disabil 2009; 34(03):275-279 